\title{
GNREL
}

\section{Nurseries Best Practices Guide for Energy and Water Efficiency, Renewable Energy, and Resilience}

Jordan Cox, Chris Hallock, Eliza Hotchkiss, Alicen Kandt, and Kosol Kiatreungwattana

\author{
National Renewable Energy Laboratory \\ Produced under direction of the U.S. Department of Agriculture (USDA) by \\ the National Renewable Energy Laboratory (NREL) under Interagency \\ Agreement IAG-15-1973.
}

NREL is a national laboratory of the U.S. Department of Energy Office of Energy Efficiency \& Renewable Energy

Operated by the Alliance for Sustainable Energy, LLC

This report is available at no cost from the National Renewable Energy Laboratory (NREL) at www.nrel.gov/publications.
Strategic Partnership Project Report NREL/TP-5R00-80131

June 2021 


\title{
GNREL
}

\section{Nurseries Best Practices Guide for Energy and Water Efficiency, Renewable Energy, and Resilience}

\author{
Jordan Cox, Chris Hallock, Eliza Hotchkiss, Alicen Kandt, \\ and Kosol Kiatreungwattana
}

National Renewable Energy Laboratory

\section{Suggested Citation}

Kandt, Alicen, Eliza Hotchkiss, Jordan Cox, Chris Hallock, and Kosol Kiatreungwattana.

2021. Nurseries Best Practices Guide for Energy and Water Efficiency, Renewable

Energy, and Resilience. Golden, CO: National Renewable Energy Laboratory.

NREL/TP-5R00-80131. https://www.nrel.gov/docs/fy21osti/80131.pdf.

NREL is a national laboratory of the U.S. Department of Energy Office of Energy Efficiency \& Renewable Energy Operated by the Alliance for Sustainable Energy, LLC

This report is available at no cost from the National Renewable Energy Laboratory (NREL) at www.nrel.gov/publications.

Contract No. DE-AC36-08GO28308
Strategic Partnership Project Report NREL/TP-5R00-80131 June 2021

National Renewable Energy Laboratory 15013 Denver West Parkway Golden, CO 80401 303-275-3000 • www.nrel.gov 


\section{NOTICE}

This work was authored by the National Renewable Energy Laboratory, operated by Alliance for Sustainable Energy, LLC, for the U.S. Department of Energy (DOE) under Contract No. DE-AC36-08GO28308. Support for the work was also provided by U.S. Department of Agriculture (USDA) under Interagency Agreement No. IAG-15-1973. The views expressed in the article do not necessarily represent the views of the DOE or the U.S. Government. The U.S. Government retains and the publisher, by accepting the article for publication, acknowledges that the U.S. Government retains a nonexclusive, paid-up, irrevocable, worldwide license to publish or reproduce the published form of this work, or allow others to do so, for U.S. Government purposes.

This report is available at no cost from the National Renewable Energy Laboratory (NREL) at www.nrel.gov/publications.

U.S. Department of Energy (DOE) reports produced after 1991 and a growing number of pre-1991 documents are available free via www.OSTI.gov.

Cover Photo: Photo from iStock, 1225625165

NREL prints on paper that contains recycled content. 


\section{Executive Summary}

This guide is a compilation of energy and water efficiency, renewable energy, and resilience best practices at United States Forest Service (USFS) nurseries and seed extractory facilities. The Forest Service Nursery System supports the agency's mission to restore and retain ecosystem health throughout its 193-million acres and ensures the future of biological diversity on federal lands.

Forest Service nurseries are agency-wide assets and have been integral to the management of National Forest resources for over 100 years. The Forest Service Nursery System previously operated 13 nurseries across the country. While only six nurseries and two standalone seed extractories remain operational today, they are vital to the health and biological diversity of federal lands, as well as public and tribal lands. ${ }^{1}$

Energy and water are critical to support the growth of about 25 million tree seedlings each year, along with native plants, such as shrubs, grasses, and wildflowers. ${ }^{2}$ USFS facilities rely on electricity and fuel to power climate control systems, lighting, computers, and appliances, as well as pumps for moving water. Water is primarily used in plant irrigation, but also in kitchen and restroom facilities. Reducing energy and water use, implementing renewable energy projects, and enhancing the resilience of nurseries is of great import to the USFS.

This guide was developed in partnership with the U.S. Department of Energy's National Renewable Energy Laboratory and is based on pilot analyses at two western nurseries.

${ }^{1}$ U.S. Forest Service, Nurseries and Seed Extractories, https://www.fs.fed.us/forestmanagement/vegetationmanagement/nurseries/index.shtml. Accessed June 1, 2021.

${ }^{2}$ U.S. Forest Service, Nurseries - Growing Future Forests, Kasten Dumroese, Rocky Mountain Research Station Research Plant Physiologist and National Nursery Specialist. May 5, 2020, https://www.fs.usda.gov/features/nurseriesgrowing-future-forests. Accessed June 1, 2021. 


\section{Table of Contents}

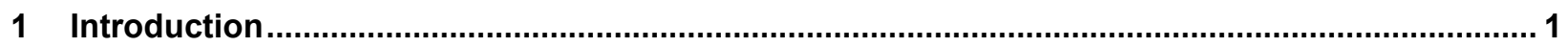

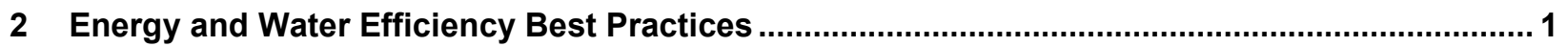

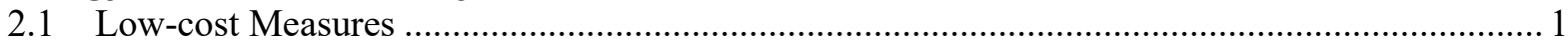

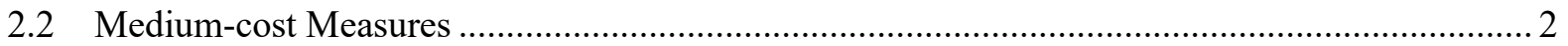

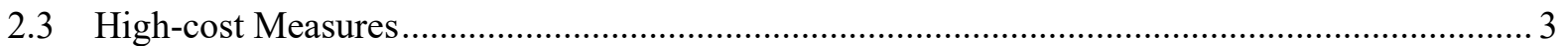

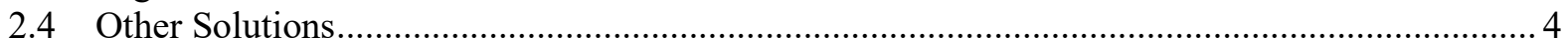

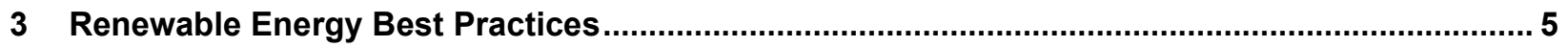

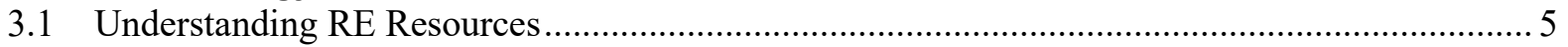

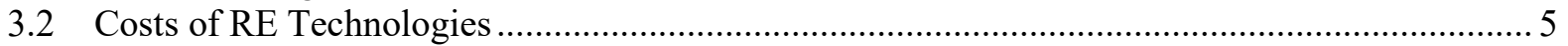

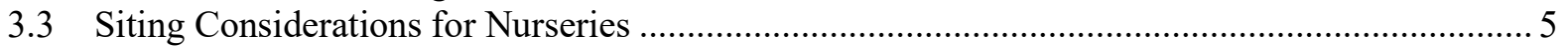

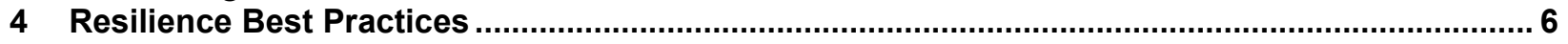

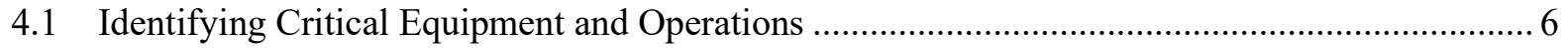

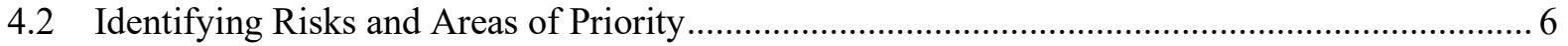

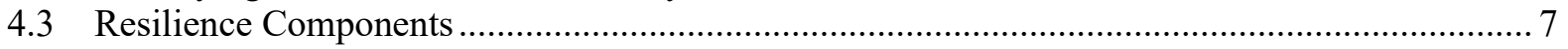

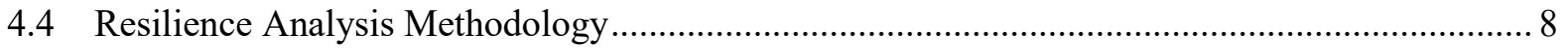

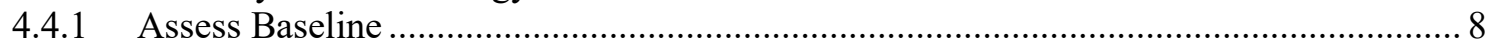

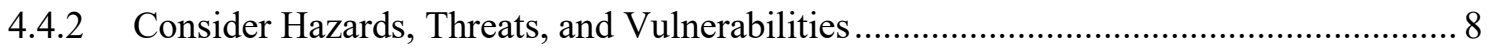

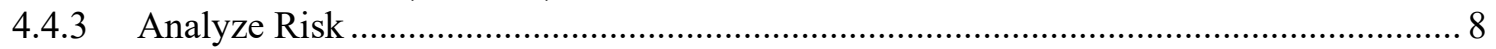

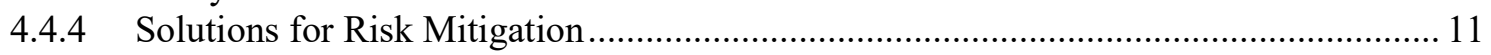

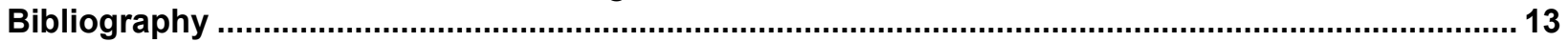




\section{List of Figures}

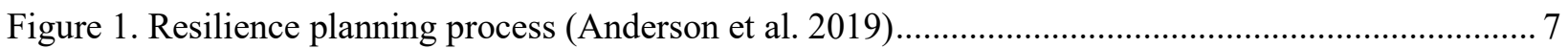

Figure 2. Example risk matrix template ........................................................................................ 10

\section{List of Tables}

Table 1. Hazard or Threat Likelihood Score Descriptions .................................................................. 9

Table 2. Vulnerability Severity Score Descriptions.................................................................... 9 


\section{Introduction}

Energy and water efficiency, renewable energy, and resilience are a high priority to the U.S. Forest Service (USFS) and have many applications within nurseries. They are also interconnected. Applying best practices in these areas has the potential to benefit nurseries through the reduction of operating costs, reducing risks, and the enhancement of the forest products being delivered to the nation.

Energy Efficiency means using less energy to perform the same task through behavior change or energy-saving technologies. Many benefits are associated with energy efficiency, including a reduction in greenhouse gas $(\mathrm{GHG})$ emissions, reduced demand for imported energy, and lower energy costs.

Water Efficiency means using less water to perform the same task through behavior change or water-saving or reuse technologies. Many benefits are associated with water efficiency, including a reduction in energy use associated with treating and moving water and the associated GHG emissions, reduced demand for treated water, and lower water costs.

Renewable Energy relates to energy generation from non-fossil fuel-based, natural sources that are constantly replenished, such as solar, wind, wave, tidal, geothermal, and biomass.

\section{Energy and Water Efficiency Best Practices}

A holistic approach to energy and water efficiency helps nurseries reduce their overall energy and water use, along with associated costs and GHG emissions. Identifying standard practices, equipment, and systems for energy and water use at nurseries provides opportunities for efficiency improvements. This section outlines solutions with the potential to conserve energy and/or water, illustrated with examples from the two pilot nurseries.

\subsection{Low-cost Measures}

1. Temperature setback: The temperature in nursery buildings is typically fixed, regardless of occupancy conditions. This can lead to wasting energy by heating and cooling buildings at times when no one is there, such as nights, holidays, and weekends. Installing programmable thermostats and implementing temperature setbacks by zone during unoccupied hours will decrease the overall cooling and heating load for the buildings at a very low cost with a short payback timeframe.

2. Weatherization: Some older nursery buildings date back to the 1960s and have multiple exterior wall and roof penetrations. Over time, degraded caulking and sealant results in heat/cooling loss from gaps around windows, doors, electrical outlets, light fixtures, and other areas. Resealing building penetrations makes heating and cooling more effective, with less wasted conditioned air, and may also increase occupant comfort. This low-cost measure has a moderate payback timeframe.

3. Irrigation schedule and duration: Setting an irrigation schedule to avoid watering during the hottest parts of the day minimizes evaporation and ensures that watering volume and frequency matches the specific needs of plants being grown. This no- to lowcost measure has a long- to moderate-payback timeframe. 
4. Reclaimed water: Reclamation takes water from a variety of sources, such as stormwater runoff, then minimally treats and reuses it for agriculture irrigation, groundwater replenishment, industrial processes, environmental restoration, and other purposes. This water is often significantly less expensive than potable water and use of reclaimed water from a local water utility can dramatically reduce potable water consumption. This low- or no-cost measure has a moderate payback timeframe.

5. Comprehensive operations and maintenance $(O \& M)$ and preventative maintenance protocols: Pragmatic O\&M programs provide operators with a diagnostic baseline, making it possible to identify possible equipment issues and solutions at early stages to optimize performance and avoid breakdowns. While O\&M plans and strategies vary by site and application, proper maintenance is essential to maximizing infrastructure efficiency, performance, and environmental and economic benefits. Preventive maintenance can optimize equipment output, prevent expensive failures, and maximize equipment lifespan. This low-cost activity has a moderate payback.

\subsection{Medium-cost Measures}

1. Light-emitting diode (LED) lighting: While the lighting in most USFS nurseries already has been updated to linear 4-foot T-8 fluorescent, high-pressure sodium (HPS), and compact fluorescent (CFL) bulbs, upgrades to LED lighting systems can deliver even greater benefits. LED systems save energy, have longer lifespans, and require less maintenance. This has a moderate payback timeframe.

2. Daylight and occupancy sensors: Spaces with windows and skylights can take advantage of natural light and minimize the need for electric lights. Daylight sensors and controls reduce the energy consumed by lighting, while maintaining adequate levels of light to conduct work and maintain safety. Installing lighting occupancy or vacancy sensors in conference and break rooms, restrooms, and large private offices can support energy efficiency. These have a short-to-moderate payback timeframe.

3. Laptop computers: Office computer equipment accounts for the primary electrical plug load in most USFS nursery facilities. Each desktop computer and monitor workstation uses approximately 300 watts, while each laptop uses approximately 45 watts. Replacing desktop computers with laptops can significantly reduce energy use while providing greater workstation flexibility. This measure has a long payback time frame; however, it can be implemented cost effectively through incremental replacement of old desktop computers as they reach the end of life or during replacement cycles.

4. Plug load timers/occupancy sensors: Occupancy sensors tied to power strips detect when an office is vacant and shut off any equipment powered by the strip, including under-cabinet light fixtures, individual heaters or fans, and desk lamps. This medium-cost measure can have a short-to-medium payback timeframe.

5. ENERGY STAR ${ }^{\circledR}$ appliances: Break rooms often have appliances, such as refrigerators, microwaves, and dishwashers. Appliances certified by the U.S. Department of Energy and U.S. Environmental Protection Agency (EPA) ENERGY STAR program use 10\%$25 \%$ less energy than noncertified models. Old appliances can be replaced cyclically at 
the end of their useful life with the ENERGY STAR units. Payback on this measure will be very low.

6. Premium efficiency motors: USFS nursery operations are generally very reliant on motors. Replacing existing electrical motors with those rated as premium efficiency models by the National Electrical Manufacturer Association (NEMA) can significantly reduce energy use. Older motors have an efficiency rating of approximately $82 \%$, compared to $92 \%-95 \%$ efficiency for NEMA premium efficiency motors. The payback timeframe for this depends on the size of the motor being replaced.

7. Low-flow fixtures: Updating old plumbing fixtures with low-flow fixtures can significantly reduce water use. While older toilets can use as much as 5 gallons of water per flush, low-flow toilets only use 1.6 gallons per flush. Older showerheads can use 3-7 gallons per minute, compared to the EPA WaterSense showerheads that use less than 2 gallons per minute. Older bathroom sink faucets have flow rates as high as 3 gallons per minute, in contrast to low-flow faucets rated with flow rates of 1.5 gallons per minute. Potential water savings depends on the building type and how water is used. This has a moderate payback timeframe but can be particularly advantageous in areas prone to drought.

\subsection{High-cost Measures}

1. Envelope insulation: Good thermal insulation not only reduces heating and cooling demands but can also contribute to improved comfort. Most of the USFS nurseries were established in the 1960s and 1970s. Although numerous additions and modifications have likely been made over the years, many building components are original and may be in poor condition. Facility exterior walls and roofs may entirely lack or have insufficient insulation. While standalone projects adding insulation to the existing envelope are likely to be expensive, integrating insulation improvements in future facility overhauls to at least meet minimum energy codes could make these updates more cost effective.

2. Highly insulated windows: Many USFS nursery buildings still have original single-pane or double-pane windows with wood or aluminum frames and no thermal breaks. Highly insulated triple- or quad-pane windows could dramatically reduce energy needed for heating and cooling, particularly for facilities located in cold climates. Window upgrades can be costly; therefore, this measure should be considered at the end of life of the original windows.

3. Replace tank water heaters with heat pump water heaters: Heat pump water heater transfers heat from one place to another to heat water using a reversed refrigeration cycle. Heat pumps water heaters are 2-3 times more efficient than electric resistance heaters. Replacing tank electric water heaters with heat pump water heaters may reduce the energy required to create hot water by $60 \%-70 \%$. This measure or the next one should be considered (not both). This has a moderate payback timeframe.

4. Replace tank water heaters with tankless water heater: Tankless (also known as instantaneous or on-demand) water heaters are another option to provide hot water only when it is needed. These water heaters eliminate stand-by losses associated with tank water heaters. A downside is that the instantaneous water heater cannot supply enough hot water for simultaneous, multiple uses. Therefore, consider hot water loads and usage 
when selecting a tankless water heater. Tankless water heaters can be electric or gas. For gas tankless water heaters, consider condensing tankless water heaters, which are even more efficient that noncondensing tankless heaters. This measure or the previous one should be considered (not both). This has a moderate payback timeframe.

5. Condensing boilers: In the two pilot nurseries, the seed extractories were heated with older boiler systems that were estimated to operate with $80 \%$ efficiency. When these old boilers are ready to be replaced, new condensing boilers can boost efficiency to $93 \%$ and reduce gas use with low-fire settings. This replacement can have a quick payback depending on the size of the boiler.

6. Split heating and air conditioning (AC) systems: Replacing older electric heaters and $\mathrm{AC}$ systems with new, efficient systems can reduce the energy used for heating by $60 \%$ and the energy used for cooling by $30 \%$. Installing a heating and AC system with a programmable thermostat allows the system to be programmed for nighttime temperature setback, further increasing the energy savings. This has a moderate payback timeframe.

7. Energy-efficient refrigeration systems: Nurseries' cold storage for seedlings and seed freezers use a large amount of electrical energy to run motors for compressors and fans. Most new indoor refrigeration units have electronically commutated motors (ECM) that can vary fan speed and reduce energy use. While the motor efficiency of older systems is approximately $82 \%$, new units offer motor efficiency of $92 \%-95 \%$. Because motors account for the greatest amount of energy used in refrigeration systems, these upgrades can result in an energy savings of $10 \%$ or more. When these systems are replaced at the end of their useful lives, this has a moderate payback timeframe.

8. Variable frequency drives (VFDs) for large pumps: VFDs controls motor speed and flow rate. Large pumps that serve a variety of loads should have a VFD. When operating multiple pumps, it is more efficient to operate more pumps at lower speeds instead of fewer pumps at higher speeds. While the cost of VFD equipment depends on motor size and could be expensive for a large motor, it also has the potential to provide significant energy savings.

\subsection{Other Solutions}

1. Commissioning: Commissioning is a quality control process that can be integrated with the installation of new systems to ensure equipment meets design and performance requirements. It is strongly recommended that commissioning be conducted after installing new equipment. An expert in the recommended systems (and advanced control strategies) should be engaged to perform commissioning to realize the greatest possible energy savings.

2. Measurement and verification $(\mathbf{M} \& \mathbf{V})$ : It is also recommended that an $M \& V$ plan following International Performance and Measurement Verification Protocol be implemented in conjunction with any major retrofit effort. M\&V plans provide ongoing energy use information for building operators to use in diagnostics and ensure the longevity of energy savings. The plan should not simply provide a one-year assessment of the retrofit's impact but provide continuous ongoing feedback on energy consumption by end use, including charting of use patterns based on utility meter data. 
3. Sustainable operations: Other sustainable operational options include sustainable purchasing, minimizing waste, recycling, and composting programs, as well as energy awareness campaigns.

\section{Renewable Energy Best Practices}

Renewable energy (RE) technologies are those that produce energy from non-fossil sources-such as solar, wind, biomass, landfill gas, ocean (including tidal, wave, current, and thermal), geothermal, municipal solid waste, or flowing water. RE systems can provide several benefits to nursery operations. These include a reduction in energy costs and emissions and, when coupled with appropriate controls and/or battery energy storage systems (BESS), an improvement in the operational resilience of a site.

The factors that determine the feasibility and cost-effectiveness of RE at a given location include the renewable resource of the site (e.g., how much sun shines on a particular location throughout a year), the cost of the energy that is being displaced by the RE, the cost of the RE technology and associated O\&M, and the availability of any incentives or rebates which could help bring down the cost of the RE technology.

\subsection{Understanding RE Resources}

Renewable energy resources vary by location. Renewable resource maps (https://www.nrel.gov/gis/data-tools.html) can be used for initial assessments of renewable resource potential at given sites. Some RE technologies, such as wind and water power systems, may warrant detailed study to quantify the renewable resource at the exact location being considered for an RE technology. Solar resources, however, are very well documented and less variable across smaller geographic areas, which often makes publicly available resource datasets sufficient for project scoping and development.

\subsection{Costs of RE Technologies}

Costs of RE technologies and BESS have fallen significantly in the past several years. The costeffectiveness of a RE system at a given location is dependent on factors including RE resource availability, technology cost, incentives, and the cost of displaced energy.

The Federal Energy Management Program (FEMP) created an interactive map (https://maps.nrel.gov/femp/) to examine the economic viability of RE technologies in the United States, factoring in potential savings from state and federal RE incentives. For a project at a specific location, the tool estimates technology costs and performance patterns, and calculates the economic value in terms of levelized cost of energy (dollars per kilowatt-hour) using geospatial site data.

\subsection{Siting Considerations for Nurseries}

Solar photovoltaic (PV) systems are one of the most prevalent types of RE systems installed within the USFS. PV systems can be installed on flat roofs, on carports, or be ground-mounted; they can even be integrated into the walls and roof of a greenhouse. PV systems are preferably sited in an area free from shade from 10 a.m. to 2 p.m., year-round, and are optimally oriented due south. Given the significant amount of land within nurseries, PV systems are often a good fit 
at these sites. Low-impact solar development (which maintains native vegetation and incorporates natural land contours rather than grading and leveling) and agrivoltaics (the dual use of land for agricultural and solar electricity production) have tremendous synergies with nursery operations. More information on these concepts can be found at https://openei.org/wiki/InSPIRE.

\section{Resilience Best Practices}

Taking a risk-informed approach to resilience planning helps nurseries understand hazards and threats that are most likely to occur, along with the potential impacts of exposing vulnerabilities within operations or systems. Identifying critical equipment and operations in the nursery and identifying the risks that are most likely to occur will help to prioritize areas for mitigation. A process for identifying critical equipment and operations and knowing how to identify risks and prioritize areas to address is outlined. Potential solutions are included to demonstrate ways to enhance resilience within nurseries, using examples from the two pilot nurseries.

\subsection{Identifying Critical Equipment and Operations}

The first step to understanding resilience needs at a nursery is determining what is essential to continuing the mission of the nursery. If the nursery's main priority is extracting, freezing, storing, and then propagating seeds, it will be essential to understand the equipment that supports each of those functions. In this example, some questions the nursery staff may need to consider are:

- Does the seed extractory have backup power that could power equipment if a grid electrical outage were to occur?

- Is the freezer or storeroom sealed and kept at an optimal temperature during a power outage? Is backup power serving the storeroom and seed freezer?

- Does the greenhouse have special heating, ventilation, and lighting requirements? Do those systems have backup power?

- Do all backup systems provide power to the critical loads for the nursery? Is there enough fuel stored onsite, or is a renewable energy and battery system installed, to prevent damaged seeds or disruption to the nursery's operations if there were a power outage for 8 hours? For 24 hours? For 3 days? For 1 week? For longer than a week?

Reviewing the needs of the critical aspects of the nursery to determine the impacts associated with a power outage or disruptions to the communications network, water supply and wastewater, and transportation network will be informative. Understanding the impacts on the nursery, along with the operational and technological solutions available at a low, medium, and high-cost range can aid in the decision-making process.

\subsection{Identifying Risks and Areas of Priority}

Pairing the essential operations and equipment with the potential risks to the nursery will help identify key areas that need to be addressed through mitigation. Risk is comprised of hazards, threats, and vulnerabilities, as described further below. Understanding how risk can impact the nursery will help in developing specific mitigation strategies that can address and reduce risk and prioritize what should be addressed first (e.g., areas of greatest risk). 


\subsection{Resilience Components}

Resilience assessment includes the following primary steps: compiling and assessing baseline information; identifying and considering hazards, threats, and vulnerabilities; analyzing risks based on potential impact and likelihood; and then developing and prioritizing mitigation actions and next steps and implementing those actions. To be most effective, this process should be continuously updated and revisited to allow for updates to the various components, such as changing missions, shifting vulnerabilities, and evolving hazards and threats; this is depicted in Figure 1.

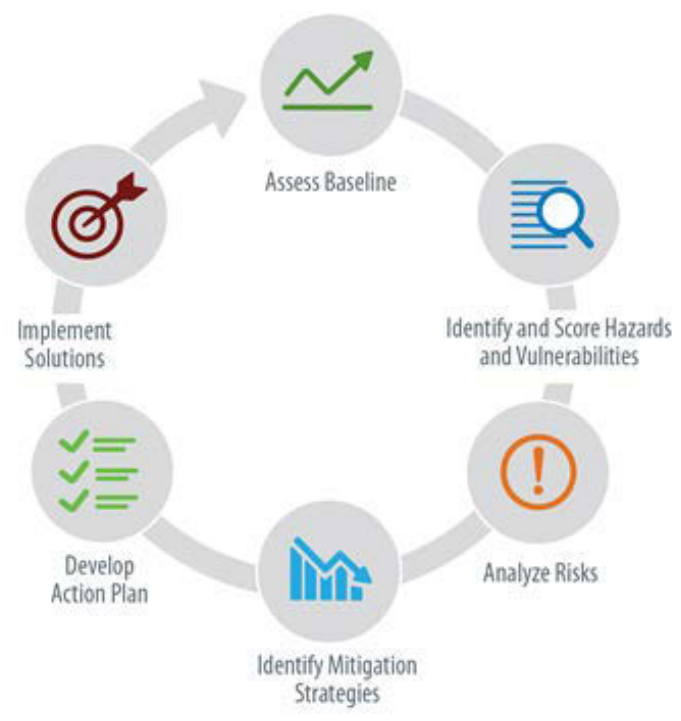

Figure 1. Resilience planning process (Anderson et al. 2019)

The baseline assessment establishes an understanding of current conditions, with a focus on understanding an agency or organization's mission, existing mitigation efforts, and environmental and socio-political contexts. Acknowledging the uncertainties, variability, and fluidity of the various components of a resilience assessment, the risk and mitigation scoring methodologies were intentionally built to be flexible. The method utilized is a qualitative and quantitative approach to estimating risk and mitigation, using stakeholder input. A more detailed approach would involve computing specific quantitative probabilities for hazards and threats and detailed cost-based consequences for vulnerabilities. However, assessment teams should be mindful of the cost-benefit of using specific probabilities and cost-based consequences.

Finally, using a framework of hazards, threats, and vulnerabilities to identify resilience challenges and, eventually, opportunities for investment and action will be useful. A vulnerability is internal to the system and/or within the site's control. These could be weaknesses within infrastructure, systems, or processes. On the other hand, hazards and threats are external and can be anything that will damage, destroy, or disrupt the site or critical assets or equipment. When taken together, the analysis should highlight where weaknesses in an existing system can be negatively exploited by a hazard or threat to have an adverse impact on the nursery. 


\subsection{Resilience Analysis Methodology}

Resilience planning is typically most effective when it can be integrated alongside existing processes and plans. In applying the resilience assessment methodology (outlined above) to the nursery - and with an eye toward developing a process that could easily be applied at other sites - the team sought to leverage existing processes for documenting and prioritizing funding for site infrastructure maintenance as part of the resilience assessment.

The resilience assessment methodology used for this analysis is derived from the National Renewable Energy Laboratory's (NREL's) Resilience Roadmap ${ }^{3}$ and Energy Resilience Assessment Methodology. ${ }^{4}$ These documents contain additional background context and guidance that may be useful to site managers and planners.

\subsubsection{Assess Baseline}

- Compile list of site facilities and assets: First, site personnel collect and consolidate information related to existing site infrastructure and assets. This information is used for the energy audit but can also identify potential site vulnerabilities.

- Review criticality: Second is to understand critical loads to the facility to frame the relative criticality across the site's assets and facilities. Criticality helps to prioritize areas for concentration and to understand the impacts to the site during a disruptive event. This information was determined by the nursery and incorporated into the analysis.

\subsubsection{Consider Hazards, Threats, and Vulnerabilities}

- Identify hazards and threats: Hazards and threats can be identified through existing assessments and stakeholder interviews. In this case, studies were collected and known hazards and threats were documented based on existing exposure analyses, past studies, and institutional knowledge. NREL discussed and identified hazards and threats with the nursery staff. Results from those discussions were incorporated in the analyses.

- Identify vulnerabilities: Vulnerabilities are weaknesses within infrastructure, systems, or processes that can, when exposed to a hazard or threat, result in damage or disruption. Vulnerabilities impact the degree of susceptibility to various hazards or threats. Similar to the process for identifying hazards and threats, known vulnerabilities were discussed based on recent experiences with disruptive events, past studies, and institutional knowledge. Interviews with site staff are invaluable when identifying vulnerabilities.

\subsubsection{Analyze Risk}

- Develop a risk matrix: After hazards, threats, and vulnerabilities were identified and grouped, the next step is to develop a risk matrix that maps hazards and threats and vulnerabilities to each other. This portrays the relationship between hazards or threats and vulnerabilities and yields a risk score for each hazard- or threat-vulnerability combination. Threats are listed on one axis and vulnerabilities on the other. The benefits

\footnotetext{
${ }^{3}$ Hotchkiss, Elizabeth L, and Alex Dane. "Resilience Roadmap: A Collaborative Approach to Multi-Jurisdictional Resilience Planning," June 26, 2019. https://doi.org/10.2172/1530716.

${ }^{4}$ Anderson, Katherine H, Elizabeth L Hotchkiss, Lisa Myers, and Sherry R Stout. "Energy Resilience Assessment Methodology," October 30, 2019. https://doi.org/10.2172/1573210.
} 
of a risk matrix are that it can be a visual representation of relationships between threats and vulnerabilities and constitute an initial prioritization of what vulnerability-hazard or threat combinations to target with solutions and foster deeper discussion with site staff. See Figure 2 for a sample template for a risk matrix.

- Score hazard or threat likelihood and vulnerability impact: Next, each hazard, threat, and vulnerability identified and included in the risk matrix was assigned a hazard or threat likelihood or vulnerability severity score. Scores were determined according to the threshold descriptions in Table 1 and 2 below. Scores can be presented either categorically (e.g., high, medium, low) or numerically (e.g., on a scale of 1-9). Numerical scores were used for this analysis.

Table 1. Hazard or Threat Likelihood Score Descriptions

\begin{tabular}{|c|c|c|}
\hline \multicolumn{2}{|c|}{$\begin{array}{l}\text { Hazard or Threat Likelihood } \\
\text { Scores } \\
\end{array}$} & \multirow[t]{2}{*}{ Threshold Descriptions } \\
\hline Categorical & Numerical & \\
\hline High & $9 / 10$ & $\begin{array}{l}\text { Almost certain to occur. Historic and frequent } \\
\text { occurrences. }\end{array}$ \\
\hline Medium-High & $7 / 8$ & More likely to occur than not. \\
\hline Medium & $5 / 6$ & May occur. \\
\hline Low-Medium & $3 / 4$ & $\begin{array}{l}\text { Slightly elevated level of occurrence. Possible, } \\
\text { but more likely not to occur. }\end{array}$ \\
\hline Low & $1 / 2$ & $\begin{array}{l}\text { Very low probability occurrence. An event has } \\
\text { the potential to occur but is still very rare. }\end{array}$ \\
\hline
\end{tabular}

Table 2. Vulnerability Severity Score Descriptions

\begin{tabular}{lcll}
$\begin{array}{l}\text { Vulnerability Severity } \\
\text { Score }\end{array}$ & & \multicolumn{1}{c}{ Threshold Descriptions } \\
\cline { 1 - 2 } Categorical & Numerical & \\
\cline { 1 - 2 } High & $9 / 10$ & $\begin{array}{l}\text { Highest magnitude of consequence. Entire power system would be } \\
\text { impacted. Extreme financial impacts would exist. }\end{array}$ \\
\cline { 1 - 2 } $\begin{array}{l}\text { Medium- } \\
\text { High }\end{array}$ & $7 / 8$ & $\begin{array}{l}\text { Significant consequences to the organization. Majority of population } \\
\text { served would be impacted. Staff tasks would be switched to } \\
\text { emergency/critical operations. Significant financial impacts would exist. }\end{array}$ \\
\cline { 1 - 2 } Medium & $5 / 6$ & $\begin{array}{l}\text { Medium magnitude of consequence. The organization would be } \\
\text { somewhat affected. Specific systems or functions would be substantially } \\
\text { interrupted, but not all. Financial impacts would be expected to change } \\
\text { budgeting plans or require reallocation of funds. }\end{array}$ \\
\cline { 1 - 2 } Low-Medium & $3 / 4$ & $\begin{array}{l}\text { Slightly elevated consequence to the organization. The power sector may } \\
\text { need to temporarily transition operations to backup systems to resolve } \\
\text { failure. Limited financial impacts may become apparent. }\end{array}$ \\
\hline Low & $1 / 2$ & $\begin{array}{l}\text { Lowest magnitude (or severity) of consequence to the organization. The } \\
\text { power sector would experience little to no effects, or an in-place backup } \\
\text { system would resolve the failure. }\end{array}$ \\
\hline
\end{tabular}

- Determine whether a relationship exists between hazards or threats and vulnerabilities (yes/no): The next step was to determine which hazards or threats were related to which vulnerabilities. Not all hazards, threats and vulnerabilities will 
necessarily intersect; not every threat will expose every vulnerability. Unrelated hazards or threats and vulnerabilities will not yield a useful risk score.

- Calculate risk score based on threat/vulnerability scores: After relationships between hazards or threats and vulnerabilities were determined, a risk score was calculated for each related hazard- or threat-vulnerability combination by multiplying the hazard or threat likelihood and vulnerability severity scores for each hazard- or threat-vulnerability combination. The result looks something like Figure 2, a heat map of where risk is highest and lowest.

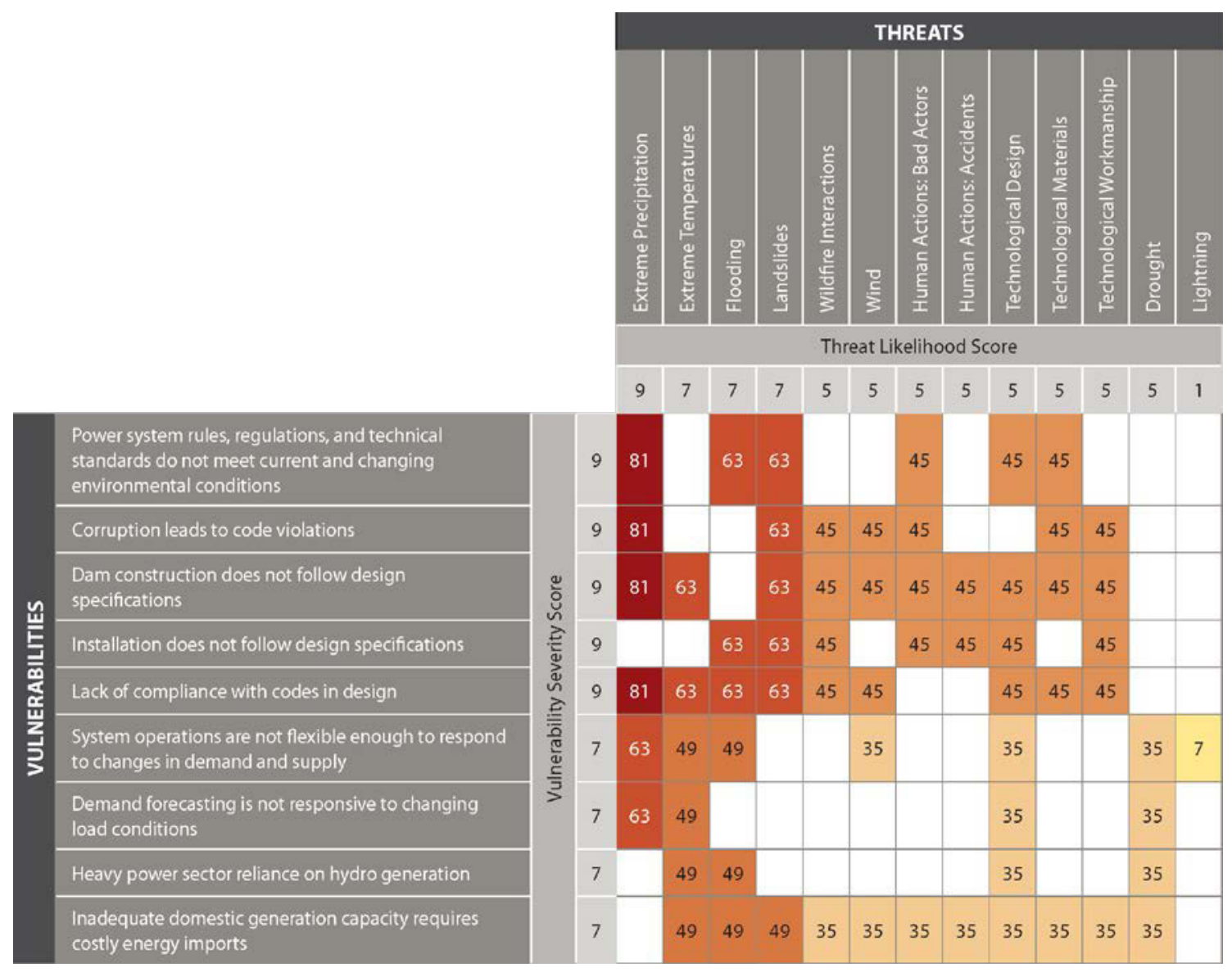

Figure 2. Example risk matrix template.

- Cross-reference and review results: Using data in the previous steps, the team determined if there were any potential differences, new gaps arising, or double counting and adjust the matrix accordingly.

The risk matrix above is a template and is unrelated to the current project. The risk matrix is a useful tool to identify solutions and then determine where those solutions apply to hazards, threats, and vulnerabilities so solutions can be prioritized and grouped for discussion about implementation. Potential solutions for risk mitigation are shown below, by infrastructure sector, to provide examples for consideration after going through a risk analysis at a nursery. 


\subsubsection{Solutions for Risk Mitigation}

After completing a risk matrix and prioritizing risk, the brainstorming and implementation of risk mitigation strategies can begin. Mitigation strategies are efforts that seek to lessen negative results of an uncontrollable hazard on the site vulnerabilities. There is no complete, exhaustive methodology for determining all potential mitigation strategies. There are, however, several lessons learned from previous work that can inform the risk mitigation process.

- Collaborating across disciplines and departments: Risk mitigation is best performed by working across expertise areas and departments so that mitigation strategies can be well-thought out and accepted by all stakeholders. Often ideas that appeal to one stakeholder might not be feasible for another and only by brainstorming together are these concerns understood and addressed.

- Accurately weighting potential solutions: Mitigation solutions can fall into many categories such as technological, operational, or organizational. A technological solution, such as installing a new heat pump, might be seem more appealing, but in fact be less cost effective than a simpler operational strategy such as manually setting temperature zones or occupancy timers in a building. When considering solutions, changes to procedure, organizational structure, or information storage should be considered alongside technology adoption.

- Identifying complementary or antagonistic solutions: Some solutions, such as redundant communications, can have complementary or synergistic affects in reducing risk. Others can address a certain type of risk while having a negative impact on other types of risk. For example, adding flood walls to one area of a campus could push flood water to other areas. Each mitigation solution should be examined for knock-on effects related to other risks.

Based on these best practices, mitigation solutions have been developed for multiple sites. While there is no one-size-fits-all solution, some examples of mitigation solutions have included:

- Communications: Adding redundant communication modes, data logging safety system failures, holding regular coordination meetings across the site to connect organizations that usually do not communicate, and safety drills that involve communicating with onsite and offsite external entities.

- Transportation: Increasing the number of site access points, increasing the amount of onsite transport fuel storage, diversifying fuel types (gasoline, diesel, electric, etc.) and personnel training.

- Water: Comparing water reserves with historical demands during extreme events, increasing on-site water storage, distributing water storage throughout a site, adding water-capturing technologies such as water filtration or rainwater capture to provide water sources, and adding water efficiency technologies. Additionally, developing a secondary water supply may be feasible for some locations.

- Energy: Diversifying on-site energy sources or energy generation technologies, implementing energy efficiency systems, designating critical loads that can be islanded from site loads, scheduling energy usage during disaster scenarios to reduce maximum draw while still serving demand, education and training of staff to understand energy usage during a disruption, redundant infrastructure, hardened infrastructure (such as 
burying power lines), hybrid energy systems for critical loads, and utilizing existing resources for energy storage (such as not opening cooler doors during a power outage). 


\section{Bibliography}

Anderson, Katherine H, Elizabeth L Hotchkiss, Lisa Myers, and Sherry R Stout. "Energy

Resilience Assessment Methodology," October 30, 2019. https://doi.org/10.2172/1573210.

Hotchkiss, Elizabeth L, and Alex Dane. "Resilience Roadmap: A Collaborative Approach to

Multi-Jurisdictional Resilience Planning,” June 26, 2019. https://doi.org/10.2172/1530716.

National Renewable Energy Laboratory, Geospatial Data Science Data and Tools, https://www.nrel.gov/gis/data-tools.html, NREL, accessed June 1, 2021.

Federal Energy Management Program (FEMP) and National Renewable Energy Laboratory, FEMP Screening Map, https://maps.nrel.gov/femp/, NREL, accessed June 1, 2021.

Federal Energy Management Program (FEMP) and National Renewable Energy Laboratory, FEMP Screening Map, https://openei.org/wiki/InSPIRE, NREL, accessed June 1, 2021.

National Renewable Energy Laboratory, Innovative Site Preparation and Impact Reductions on the Environment (INSPIRE), https://openei.org/wiki/InSPIRE, NREL, accessed June 1, 2021. 